\title{
IoT based Biotelemetry for Smart Health Care Monitoring System
}

\author{
Vivekanadam Balasubramaniam, \\ Faculty of Computer Science and Multimedia, \\ Lincoln University College, \\ Kota Bharu, Malaysia \\ E-mail: vivekanandam@lincoln.edu.my
}

\begin{abstract}
The concept of biotelemetry evolved to assess the physiological data of a person under normal circumstances without obstruction to the patient. It allows evaluation of risk factors influence the person health on their daily activities. Recent technology development enhances the features of biotelemetry as wireless applications which allows the physician to monitor the patient health remotely. Biotelemetry obtains great values in hospitals by continues monitoring process as it reduces the burden to physician regular checkups. ECG telemetry is one of the predominant biotelemetry application employed to monitor the heart rate and arrhythmias. Proposed research work focusses the key features of ECG telemetry and provides an internet of things (IoT) based application to monitor the patient health in an indoor and outdoor environment. Along with medical terms, data management parameters are analyzed in the experimental section to emphasize the proposed work performance.
\end{abstract}

Keywords: - Electrocardiogram (ECG), Biotelemetry, Internet of Things (IoT)

\section{Introduction}

Electrocardiogram (ECG) is one of the most important tools for diagnosing cardiac diseases. It measures and records the activities of the heart through the electrodes that are placed on the skin. Telemetry is a device for continuous monitoring of the patient ECG, oxygen saturation, and respiratory rate of a patient and transmits the information through the wireless network and stores on the central server for monitoring. The accuracy of telemetry is depending on the preparation of the skin, lead and electrode placement, maintenance of the equipment, and monitoring the patients Telemetry functions with 5 lead such as White, Red, Brown, Green, and Black. Each color is used for different purposes. For setting up the telemetry, first to ensure the functionality of the battery level and attachments leads to the patients. Then attach the lead wires to the telemetry. Then arranging the telemetry from the central monitor and make sure it must be displayed on the bedside monitor. For setting up the monitor, there will be two leads such as lead 1 is used for diagnosing the QRS complex, and lead 2 is used for diagnosing and monitoring the heart rate and atrial activity. This telemetry can be viewed from the central monitor and in each pod, there will be a monitor and also at the bedside monitor. Finally, adjust the settings of ECG. It is necessary to maintain the telemetry device by checking the battery condition at regular intervals of time and the device itself also will intimate by alarm for charging the batteries. Then the electrodes should be changed regularly. Figure 1 depicts the ECG monitoring process in detail. 
Journal of Information Technology and Digital World (2020)

Vol.02/ No. 03

Pages: 183-190

https://www.irojournals.com/itdw/

DOI: https://doi.org/10.36548/jitdw.2020.3.006

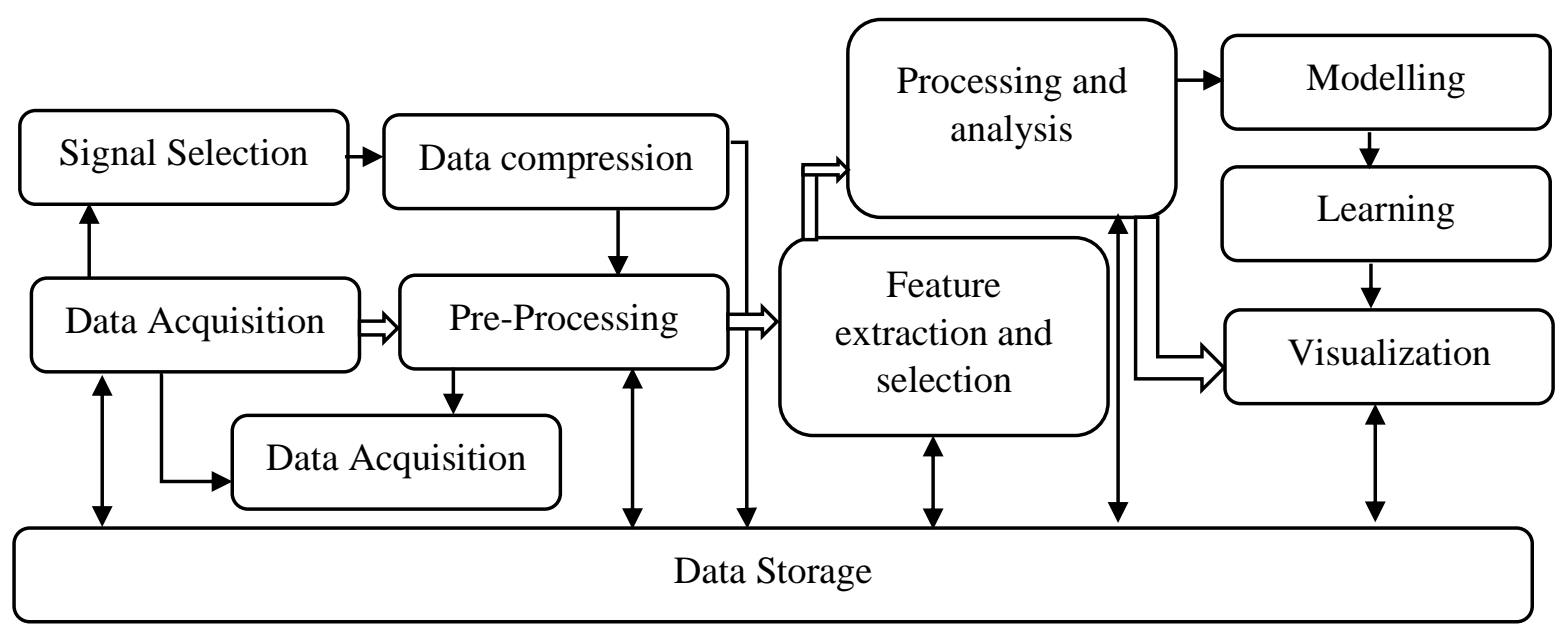

Fig. 1 ECG Monitoring process

Telemetry ECG devices provide fast and easy ECG monitoring for the patients. It is important to choose a device with good working conditions, signal quality, and cost of the device. Multi-purpose ECG telemetry device is mainly composed of ECG device and comfortable mobile applications for the patients and the physicians. Then a cloud-based analysis pipeline is needed. This method allows the patients to record the short-term ECG signals easily in the post-event by utilizing the dry electrodes without any prior arrangements. But in some situations, patients preferred to use the long-term signals in two modes such as holter and loop modes by using the wet electrodes. To solve the signal quality fluctuation problem, cause due to the usage of different electrodes types and different placements on the patient's chest. For each of the different modes, there will be a customized ECG signal processing and interpretation pipeline.

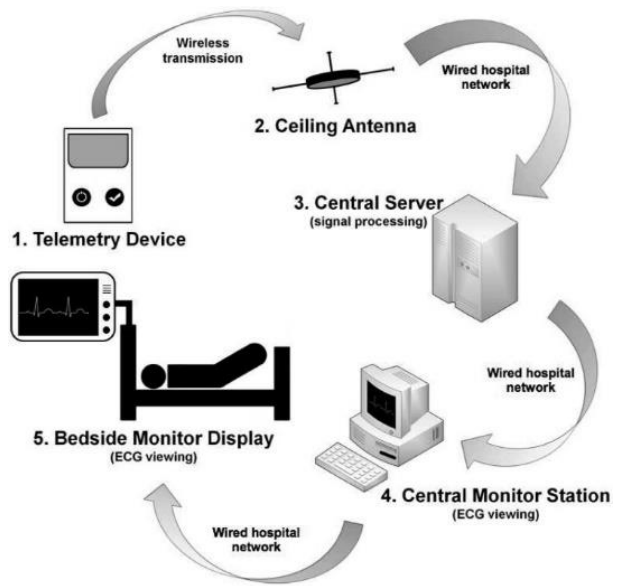

Fig. 2 ECG Telemetry System

The result of the system will give a short-term recorder design. It was performed under 391 patients with 12 leads golden standard ECG. It also consists of a multi-purpose ECG telemetry device that allows the physicians to select the desirable working mode in the device according to the patient's condition. It also helps to manage the working modes including the technical requirements. Figure 2 depicts the ECG Telemetry System. It provides reliability with three leads for the physicians with the direct symptom-rhythm correlation. This design consists of DSP algorithms/ storage, user applications, transmitting information, and cloud-based analysis. In this method, it allows the patients to record their ECG signals and send to the algorithm/storage locations. One can have access to the signals

Information Technology $\&$ Digital World 
Journal of Information Technology and Digital World (2020)

Vol.02/ No. 03

Pages: 183-190

https://www.irojournals.com/itdw/

DOI: https://doi.org/10.36548/jitdw.2020.3.006

that are sent with the help of mobile and web applications. So, they can view all the signals which have been sent. It is recorded and sent through Bluetooth to mobile application using the GSM network that is embedded in the user device and it can communicate directly with the corresponding server using the network. Using this method, almost $96 \%$ of patients recorded and transmitted their ECG signals of different age groups.

\section{Related Works}

A vast survey has been made in view of ECG telemetry to obtain the merits and demerits of existing research models. The role of wireless data transmission in telemetry is reported by Pyungwoo Yeon et.al [1] to observe the brain signals of a patient. Using inductive link with free floating neural probe across the brain the telemetry system is achieved. Inductive links are shared with coil links for effective wireless transmission and improves the efficiency of the system in terms of data absorption rate with minimum bit error rate. Preclinical risk assessment using multiple channel options is reported in Christopher Regan et.al [2] research model. Research work focused to develop vivo tools to obtain clinical assessment of ECG signals using multiple cardiac axes and magnitude leads. Proposed telemetry system observes and simultaneously records the data such as cordial ECG and arterial blood pressure. These collected information is processed through ECG analysis models to obtain better assessment results to diagnose the issues in patients. Combined approach enhanced the assessments results and provides better performance.

Yaël Kolasa et.al [3] reported wireless telemetry application in clinical studies and its opportunities to improve the reliability of the monitoring system along with accuracy. The challenges in wearable devices and operational facts are identified and discussed in research work and proposed a silico-based model for effective control over ECG signals in telemetry systems. Considering the features of telemetry systems such as packet received ratio, battery lifetime and distortion in ECG signals proposed model evaluated the efficiency of the system. Further it is optimized based on central composite model which further improves the quality of services in ECG telemetry. A fully implanted telemetry system is reported in Joseph et.al [4] research model for long term monitoring. Proposed model considers he subcutaneous glucose level to measure the diabetes range using membrane sensors and oxygen electrodes. For a maximum duration of 180 days is measured with $2 \mathrm{~min}$ time interval the proposed model monitors the glucose level of patients. Tissue glucose and blood glucose levels are observed and provides statistical results to diagnose the patient if the range exceeds the threshold.

Passive phase shift keying for data transmission from medical implants is reported by Dai Jiang et.al [5]. Research work focuses the modulator design to improve the data transmission range using inductive link. Proposed approach attains $13.56 \mathrm{MHz}$ of frequency range with data rate of $1.35 \mathrm{Mbps}$ which suits for biomedical implant devices. Low coupling coefficients and high-power demand are the key features of the proposed telemetry system. Similar model is reported in Hanjun Jiang et.al [6] research work which further improves the frequency range into 400-450MHz using a 10Mbps OQPSK transceiver in telemetry operation. Proposed approach attains high data rate and minimum power consumption by combining the lookup table and uncorrelated DC offset calibration models. This combined approach enhances the system performance in transmission and reception at $10 \mathrm{Mbps}$ data rates.

Pongphan Leelatien et.al [7] proposed a real time monitoring system to transmit biological data after liver transplant to manage the patient health status. Wireless communication established from the liver region through implanted devices. Using ultra-wideband communication, the status of patient is monitored and if there are abnormalities identified suitable drugs are preferred to ensure the patient health from specific location. proposed approach used UWB for communication which offers better wireless communication system utilization. Xiang Gao et.al [8] reported the issues in UWB telemetry application through conformal balloon antenna. Conformal conductive strips are used along with biconical feeding structure which provides shield protection and enhances the resistance. Proposed antenna produces omnidirectional pattens with flat gain is the design limitations.

Bio matched horn antenna for lossless communication in bio implants is reported in John Blauert et.al [9] research model. With $2.4 \mathrm{GHz}$ of wireless bandwidth the proposed model attains better performance in terms of data rate and bit error rate. The horn antenna is placed under the tissue with water filled holes to replicate the frequency dependent

ISSN: 2582-418X

Submitted: 17.08 .2020

Accepted: 11.09 .2020

Published: 18.09 .2020

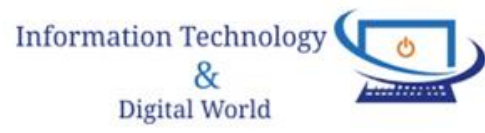


Journal of Information Technology and Digital World (2020)

Vol.02/ No. 03

Pages: 183-190

https://www.irojournals.com/itdw/

DOI: https://doi.org/10.36548/jitdw.2020.3.006

parameters. Proposed model attains minimum transmission loss and improved communication range. A planar inverted-F antenna design is reported in Xian-Tao Yang et.al [10] research model for effective monitoring in an indoor environment. Proposed telemetry system has reconfigurable features in its antenna design which reduces the multipath reflection and mismatch in the transmission environment. Two PIN diodes are used as groups to switch the radio frequency in $x-y$ directions and orthogonal polarization is used to reduce the link failures in the network. Proposed model attains better coefficient of $-10 \mathrm{~dB}$ which is considered as better coefficient value than similar antenna designs.

Abhishek et.al [11] proposed a low power telemetry system with $401-406 \mathrm{MHz}$ frequency band for health care applications. The essential condition for telemetry system and bio signal communication range are considered in the design process and developed a low power reliable wireless communication implant module. This transceiver model attains $-18 \mathrm{dBm}$ of output power with $-75 \mathrm{dBm}$ of sensitivity which is suitable for electrocardiogram and photoplethysmogram signals. Proposed model attains a maximum communication range of $3 \mathrm{~m}$ with minimum bit error rate which is quite useful for biomedical applications. Optimizing telemetry system to improve the wireless range is reported in John et.al [12] research model. Proposed research work discusses the features of implant devices and developed an active implant device with $2 \mathrm{MHz}$ range wireless system works with $420 \mathrm{kHz}$ frequency. Experimentally work of proposed research attains better performance in wireless range for data transmission.

IoT enabled ECG telemetry system is reported in Udit Satija et.al [13] research work which observes the cardiac health of patients in an indoor environment. Using three different modules such as ECG sensing module, signal quality aware module and signal quality assessment module the proposed telemetry system is developed for automatic classification. Based on different physical activities the proposed model is experimentally verified through machine learning approach and validate the features of ECG signals. Better reliability and improved accuracy are the key advantages of the proposed research model. Jinhong Guo et.al [14] proposed an IoT based medical application for digital services to manage medical images, biophysical parameters and biological information. Since the acquisition and transmission of data through wireless channel requires wide bandwidth and better speed. Proposed work considers these facts and developed an application model using mobile devices to acquire data from patients through a dongle. This electrochemical analyzer measures the blood glucose, uric acid levels and sent the information to the physician for further decisions. Proposed model is experimentally verified and the results are compared with conventional clinical biochemical analyzer. Though the performance of proposed model is better, but the cost for smart analyzer is the major limitation of the system.

Philip et.al [15] proposed an advanced IoT application for remote health monitoring. Biomedical strip-based diagnosis system provides personalized monitoring environment and long-range analysis for urinary tract infections. With path loss of 119 to $141 \mathrm{~dB}$ proposed model attains the communication range maximum of $6 \mathrm{Km}$ which provides better data communication to telemetry system. Mohsen Hooshmand et.al [16] proposed a fitness monitor system to measure blood pressure, ECG, respiration. Considering the facts in biomedical devices such as battery power, hardware constraint, memory and energy management a subject adaptive unsupervised signal compressor is proposed in the research model. Proposed model reduces the energy consumption through lossy compression algorithm and enhances the battery life in biomedical devices. From the survey it is observed that energy management, communication range, reliability and accuracy are the major parameters considered in telemetry systems. Considering these issues, an integrated IoT system is proposed in this research work for better data transmission and improved accuracy, efficiency in ECG telemetry systems.

\section{Proposed work}

The proposed ECG telemetry system consists of ECG device to capture the signals, an algorithm to route the collected information from terminal to server and classification module to analyze the collected information. It helps the physicians to access the patient information using application and immediate attention are notified through alert systems. Sensors are framed to constitute the IoT environment and wireless electrodes are interlinked with sensors to transmit the data from patient to transmitter section. Observed information is further amplified and transmitted to cloud and classification is performed using artificial neural network module. The resultant classified data and decisions

186

ISSN: $2582-418 \mathrm{X}$

Submitted: 17.08 .2020

Accepted: 11.09.2020

Published: 18.09.2020

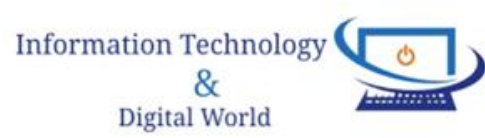


Journal of Information Technology and Digital World (2020)

Vol.02/ No. 03

Pages: 183-190

https://www.irojournals.com/itdw/

DOI: https://doi.org/10.36548/jitdw.2020.3.006

are forwarded to the physician for further process. Figure 3 depicts the overall process flow of proposed telemetry system.

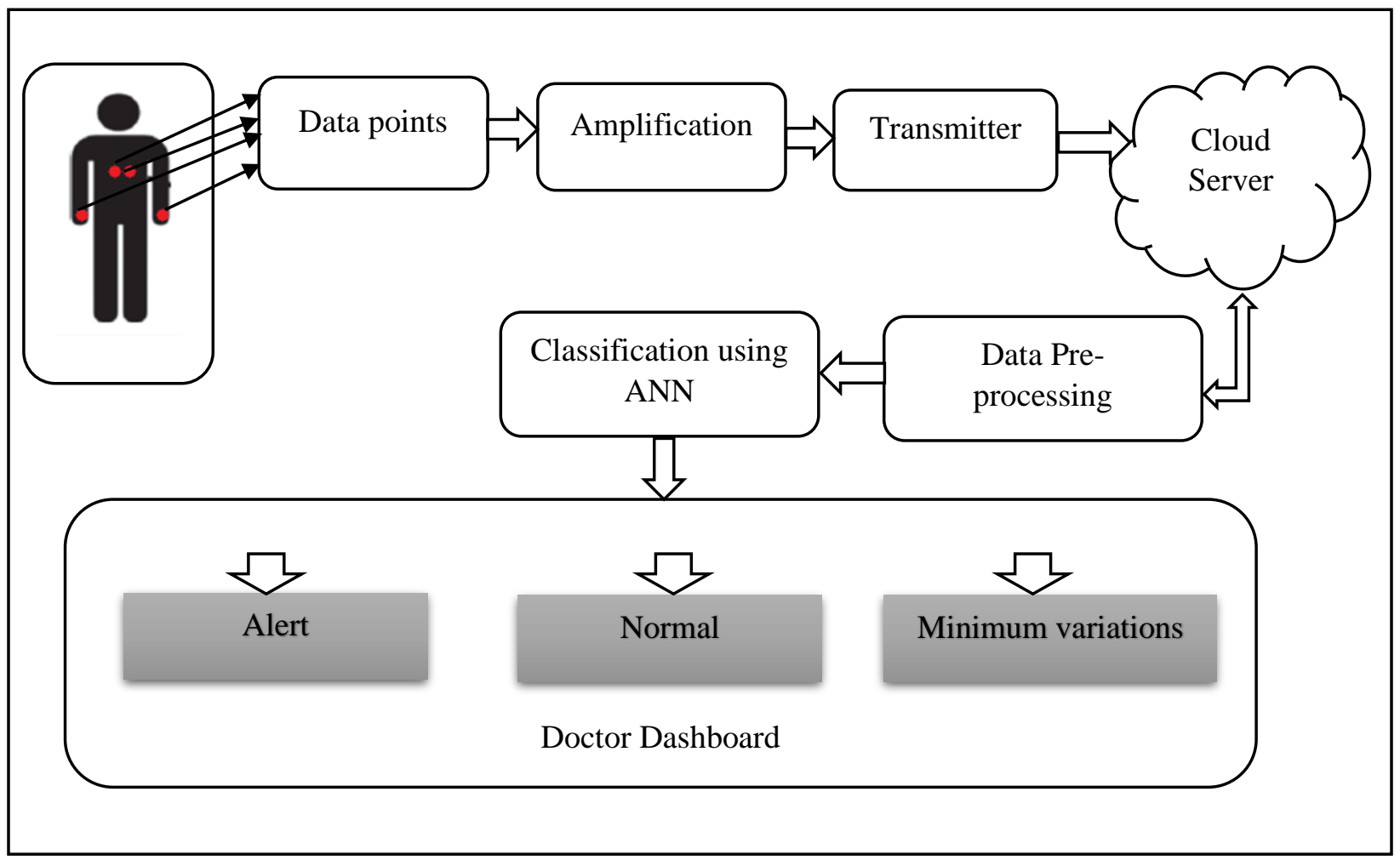

Fig. 3 Proposed IoT ECG Telemetry System

Detection of signals is an important process in ECG signal analysis. The temporal information is observed for evert beat and it is classified using neural network algorithm. Most of the conventional models uses template matching and non-linear transformations to validate the classification process. However, the results of such models are not effective. Upon classifying the ECG signal using machine learning models, the average heart rate can be computed by analyzing the heart beats for single time period. Also, ECG telemetry system has option to detect arrhythmia, segment changes, duration of atrial fibrillation and number of episodes. So, it is important to transmit the data effectively in telemetry systems and classification should be precise to obtain clinical support. Similarly, transmission medium is important for a telemetry system. The system should cover maximum range of data transmission to attain better efficiency. Various medium such as Bluetooth, ZigBee and Wi-Fi modules are used in data transmission in an indoor environment. A comparison of ECG sensing network is given in table 1.

Table 1. ECG Sensing Networks

\begin{tabular}{|c|c|c|c|c|}
\hline S.No & Standards & Bluetooth Module & Zig Bee Module & Wi-Fi Module \\
\hline 1 & Data Rate & $3-24 \mathrm{Mbps}$ & $10-250 \mathrm{Kbps}$ & $11-54 \mathrm{Mbps}$ \\
\hline 2 & Range & Maximum 30m & Maximum 20m & Maximum 200m \\
\hline 3 & Power Consumption & Low & Low & Medium \\
\hline 4 & Terminal dependency & Dependent & Dependent & Independent \\
\hline 5 & Protocol & IEEE 802.15 .1 & IEEE 802.15.4 & IEEE 802.11 \\
\hline
\end{tabular}


Journal of Information Technology and Digital World (2020)

Vol.02/ No. 03

Pages: 183-190

https://www.irojournals.com/itdw/

DOI: https://doi.org/10.36548/jitdw.2020.3.006

The ECG acquisition model has event recorder which performs long term and short-term event recording process to sense the ECG signal. Sensors connected with dry electrodes monitors the ECG signals without any wired connections. The important features affecting the ECG signals are noise factors and skin impedance. The stability of the electrodes is need to be maintained in order to obtain realistic data. Proposed design used $\mathrm{AgCl}$ electrodes in the device to sense the data as long-term recording.

Initially the signals are collected using the device and an amplifier is used to amplify the received signal. wireless transmission is performed in the system, so an analog to digital converter is used to convert the collected information and then it is processed into the cloud server for further analysis. The collected information is huge and frequency of data collection will be every five minutes. In order to manage space issues cloud server is introduced in telemetry system and then the essential features are identified and classified using artificial neural network model. The classified results are updated in the doctor dashboard with three categories as alert, normal and minimum variation. Once the alert is flagged in the dashboard, an immediate attention is required for the patient, minimum variation describes the variation in signal level and needs necessary medication to maintain the level. Normal doesn't require any action and the monitoring process can continue with the present status.

\section{Result and Discussion}

The proposed telemetry system is validated through a clinical analysis. 400 patients are considered for evaluation at different time intervals. The experimentation is conducted in indoor and outdoor environments where the elders are placed in the indoor environment and age group of 25-40 are placed in the random positions. Among then 200 males, 150 are with age group of 25-40 and 50 members are above 40 years old. Similarly, 200 female candidates are selected, 164 are age group of 25-40 and others are above years old. The evaluation process is validated in two steps as before and after telemetry. Initially using conventional ECG analysis, the respective data are noted and then proposed designbased data collection and analysis is performed. Table 2 depicts the signal quality of the candidates

Table 2 Average Signal Quality

\begin{tabular}{|c|c|c|c|}
\hline S.No & Population & Age range & Signal Quality \\
\hline 1 & Adult Male & $25-40$ & $4.03 \pm 0.20$ \\
\hline 2 & Adult Male & $>40$ & $4.19 \pm 0.20$ \\
\hline 3 & Adult Female & $25-40$ & $4.12 \pm 0.20$ \\
\hline 4 & Adult Female & $>40$ & $4.20 \pm 0.20$ \\
\hline
\end{tabular}

From the average signal quality, it is observed that the quantified age group is $>40$ and the correlation coefficients are need to be related to validate the results. The correlation coefficient for the two age groups are depicted in table 3 .

Table 3. Correlation Coefficients

\begin{tabular}{|c|c|c|c|}
\hline S.No & Population & Age range & Correlation Coefficient \\
\hline 1 & Adult Male & $>40$ & 0.8820 \\
\hline 2 & Adult Female & $>40$ & 0.8915 \\
\hline
\end{tabular}

To evaluate the ECG signal quality of patients the average signal quantity is measured and compared with 12 ECG leads golden standard. In order to understand the correlation coefficient, the possible distortions and electrodes conditions are considered. Figure 4 depicts the observed ECG signal with proposed model and golden standard device. It is observed that proposed system has ability to measure the signals at various angles similar to golden standard devices. The improved performance of proposed system is achieved through the sensor at electrodes which effectively captures the signals and transmits to the classification module. 
Journal of Information Technology and Digital World (2020)

Vol.02/ No. 03

Pages: 183-190

https://www.irojournals.com/itdw/

DOI: https://doi.org/10.36548/jitdw.2020.3.006

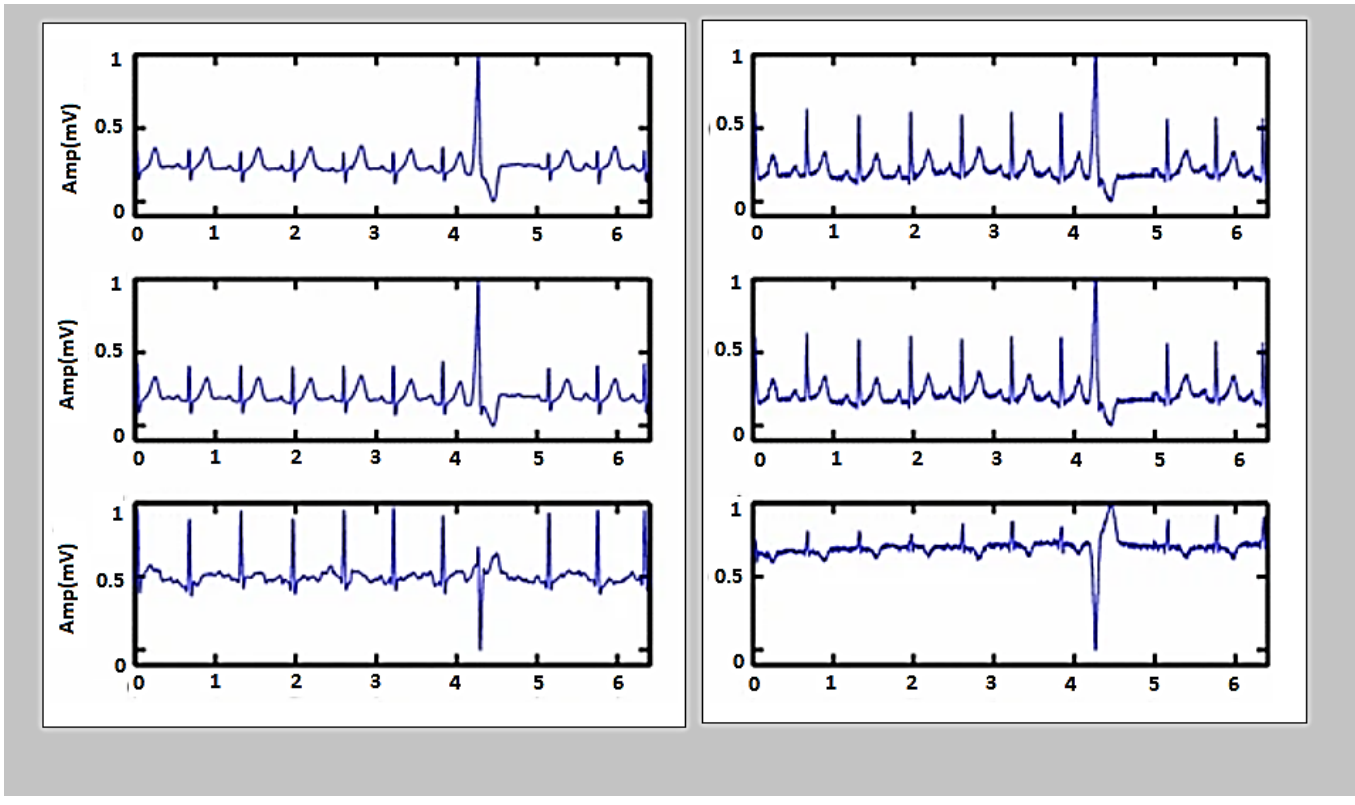

(a)

(b)

Fig. 4 Observed ECG signal (a) proposed model (b) Golden standard device

The performance of proposed telemetry system is further validated by analyzing the positive prediction value and sensitivity for classification and detection process. Table 4 depicts the values for proposed model and golden standard model.

Table 4 Performance Comparison

\begin{tabular}{|c|c|c|c|c|c|c|c|c|}
\hline \multirow{2}{*}{ Parameters } & \multicolumn{4}{|c|}{ Golden standard } & \multicolumn{4}{c|}{ Proposed model } \\
\cline { 2 - 9 } & $\begin{array}{c}\mathrm{M} \\
25-40\end{array}$ & $\begin{array}{c}\mathrm{M} \\
>40\end{array}$ & $\begin{array}{c}\mathrm{F} \\
25-40\end{array}$ & $\begin{array}{c}\mathrm{F} \\
>40\end{array}$ & $\begin{array}{c}\mathrm{M} \\
25-40\end{array}$ & $\begin{array}{c}\mathrm{M} \\
>40\end{array}$ & $\mathrm{~F}$ & $\mathrm{~F}$ \\
$25-40$ & $>40$ \\
\hline Positive Prediction Value (\%) & 94.55 & 95.41 & 95.52 & 94.99 & 97.14 & 97.26 & 97.32 & 97.44 \\
\hline Sensitivity (\%) & 96.25 & 96.64 & 96.54 & 96.43 & 98.22 & 98.84 & 98.43 & 98.88 \\
\hline
\end{tabular}

It is observed from the table the proposed model attains better positive prediction value on an average of 97 $\%$ which is $4 \%$ greater and in terms of sensitivity proposed model attains $98 \%$ average which is $2 \%$ greater than golden standard model. The positive prediction and sensitivity of proposed system increases due to its effective data transfer, data management and classification characteristics. Consequently, the complexity of the system is also reduced using wireless system and automatic classification of results reduces the work burden to the physician.

\section{Conclusion}

An Internet of Things (IoT) based biotelemetry is proposed in this research work as smart monitoring by analyzing ECG signals of a patient. Telemetry is an important feature in smart monitoring systems. Data management and data transfer is enabled in the telemetry through IoT module and collected data is stored in cloud to improve the quality of services. Further the information is classified using artificial neural network module to obtain clinical decision which supports the physician to provide immediate medication. Standard golden procedure of ECG analysis is compared 
Journal of Information Technology and Digital World (2020)

Vol.02/ No. 03

Pages: 183-190

https://www.irojournals.com/itdw/

DOI: https://doi.org/10.36548/jitdw.2020.3.006

with the proposed telemetry system and it is observed that proposed model is better than conventional model in terms of positive prediction value and sensitivity. Further this research could be improved using optimization models to enhance the network parameters.

\section{References}

1. Pyungwoo Yeon, S. Abdollah Mirbozorgi, Jaemyung Lim, Maysam Ghovanloo (2017). Feasibility Study on Active Back Telemetry and Power Transmission Through an Inductive Link for Millimeter-Sized Biomedical Implants. IEEE Transactions on Biomedical Circuits and Systems. 11(6): 1366-1376.

2. Christopher Regan, Tom Delahanty, Robert Brockway, Patrick Fanelli, Frederick Sannajust (2019). Evaluation of clinically-relevant proarrhythmic ECG biomarkers in conscious beagle dogs using a newly developed multiple lead ECG telemetry implant. Journal of Pharmacological and Toxicological Methods. 99:1-15.

3. Yaël Kolasa, Jean-Philippe Georges, Thierry Bastogne (2019). Computer-aided design of ECG telemetry systems for online cardiac monitoring. Journal of Pharmacological and Toxicological Methods. 99:26-33

4. Joseph Y. Lucisano, Timothy L. Routh, Joe T. Lin, David A. Gough (2017). Glucose Monitoring in Individuals with Diabetes Using a Long-Term Implanted Sensor/Telemetry System and Model. IEEE Transactions on Biomedical Engineering. 64(9): 1982-1993.

5. Dai Jiang, Dominik Cirmirakis, Matthew Schormans, Timothy A. Perkins, Nick Donaldson, Andreas Demosthenous (2017). An Integrated Passive Phase-Shift Keying Modulator for Biomedical Implants With Power Telemetry Over a Single Inductive Link. IEEE Transactions on Biomedical Circuits and Systems. 11(1): 64-77.

6. Hanjun Jiang, Zhaoyang Weng, Yang Li, Jingjing Dong, Woogeun Rhee, Zhihua Wang (2016). 10 Mbps 0.3 nJ/bit OQPSK transceiver IC for 400-450 MHz medical telemetry. Electronics Letters. 52(22): 1830-1832.

7. Pongphan Leelatien, Koichi Ito, Kazuyuki Saito, Manmohan Sharma, Akram Alomainy (2018). Channel Characteristics and Wireless Telemetry Performance of Transplanted Organ Monitoring System Using Ultrawideband Communication. IEEE Journal of Electromagnetics, RF and Microwaves in Medicine and Biology. 2(2): 94-101.

8. Xiang Gao, Zhenghui Xia, Shilong Wang, Jia Du, Yangyi Sui (2017). Low-Cost Wind-Insensitive Ultrawideband Balloon Antenna for Seismic Telemetry Systems. IEEE Antennas and Wireless Propagation Letters. 16: 28322835.

9. John Blauert, Asimina Kiourti (2019). Bio-Matched Horn: A Novel 1-9 GHz On-Body Antenna for Low-Loss Biomedical Telemetry with Implants. IEEE Transactions on Antennas and Propagation. 67(8): 5054-5062.

10. Xian-Tao Yang, Hang Wong, Jun Xiang (2019). Polarization Reconfigurable Planar Inverted-F Antenna for Implantable Telemetry Applications. IEEE Access. 7: 141900-141909.

11. Abhishek Srivastava, Nithin Sankar K, Baibhab Chatterjee, Devarshi Das, Meraj Ahmad, Rakesh Keshava Kukkundoor, Vivek Saraf, Jayachandran Ananthapadmanabhan, Dinesh Kumar Sharma, Maryam Shojaei Baghini (2018). Bio-WiTel: A Low-Power Integrated Wireless Telemetry System for Healthcare Applications in 401-406 MHz Band of MedRadio Spectrum. IEEE Journal of Biomedical and Health Informatics. 22(2): 483494.

12. John.B, Spink.C, M. Braunschweig, R. Ranjan, D. Schroeder, A. Koops, G. Woldt, G. Adam, W.H. Krautschneider (2018). Optimisation of inductive telemetry links in an implantable medical device for a wireless range of $25 \mathrm{~cm}$. Electronics Letters. 54(23): 1315-1316.

13. Udit Satija, Barathram Ramkumar, M. Sabarimalai Manikandan (2017). Real-Time Signal Quality-Aware ECG Telemetry System for IoT-Based Health Care Monitoring. IEEE Internet of Things Journal. 4(3): 815-823.

14. Jinhong Guo (2018). Smartphone-Powered Electrochemical Biosensing Dongle for Emerging Medical IoTs Application. IEEE Transactions on Industrial Informatics.14(6): 2592-2597.

15. Philip A. Catherwood, David Steele, Mike Little, Stephen Mccomb, James Mclaughlin (2018). A CommunityBased IoT Personalized Wireless Healthcare Solution Trial. IEEE Journal of Translational Engineering in Health and Medicine. 6: 1-13.

16. Mohsen Hooshmand ,Davide Zordan, Tommaso Melodia, Michele Rossi (2017). SURF: Subject-Adaptive Unsupervised ECG Signal Compression for Wearable Fitness Monitors. IEEE Access. 5: 19517-19535. 